\title{
Un análisis del concepto lockeano de "pueblo" a partir del consentimiento y la resistencia
}

\author{
Martín OLIVEIRA \\ Universidad de Buenos Aires - CONICET
}

\begin{abstract}
RESUMEN
Este trabajo intenta reconstruir el concepto de "pueblo" en la teoría política de John Locke a partir de su teoría del consentimiento. En primer lugar analizamos la noción de "resistencia política" que arroja importantes elementos para caracterizar al pueblo lockeano. Posteriormente complementamos dicho enfoque con la hipótesis de que la distinción entre consentimiento expreso y tácito es clave para determinar quién forma parte del pueblo, mientras que intentaremos mostrar que los análisis de esta distinción centrados sólo en el deber de obedecer las leyes se muestran insuficientes.
\end{abstract}

PALABRAS CLAVE: Locke, representación, pueblo, resistencia, consentimiento

\begin{abstract}
This paper aims to reconstruct the concept of "people" in the political theory of John Locke considering his theory of consent. First, we analyze the notion of "political resistance" that provides important elements to characterize the Lockean people. We then complement that perspective with the hypothesis that the distinction between express and tacit consent is key to determine who can be part of the people, as we try to demonstrate that the analysis of this distinction solely focused on the duty to obey the law turns out to be insufficient.
\end{abstract}

KEYWORDS: Locke, representation, people, resistance, consent

\section{Introducción}

En este trabajo indagaremos la noción lockeana de pueblo a partir de su teoría del consentimiento. Dado que es el pueblo, cuando cada individuo abandona el estado de naturaleza y se une a la sociedad civil, el que constituye el poder político pero también el que se resiste a éste cuando sufre alteraciones o viola su confianza, resulta de enorme importancia determinar quiénes pueden formar parte de este cuerpo político. Bajo la hipótesis de que la distinción entre consentimiento expreso y tácito es clave para determinar quién forma parte del pueblo, intentaremos mostrar que los análisis de la distinción centrados sólo en el deber de obedecer las leyes se muestran insuficientes. Asimismo buscaremos reparar en que, para obtener una perspectiva más rica de la teoría lockeana, debemos atender a otros tipos de deberes que conlleva la membrecía al Estado.

Dedicaremos un primer apartado apartado, en consecuencia, a desarrollar la teoría lockeana de la constitución de la sociedad civil y el pueblo, así como su doctrina de la resistencia civil. Posteriormente evaluaremos dos análisis clásicos del argumento del 
consentimiento centrados en el deber de obediencia política con el objeto de mostrar que, para echar luz sobre el concepto lockeano de pueblo, debemos detenernos también en otros tipos de deberes que genera la membrecía al Estado. A continuación intentaremos determinar cómo estos deberes aportan matices a la interpretación del concepto de pueblo para, finalmente, ofrecer unas breves conclusiones al respecto.

\section{El problema de la resistencia y su relación con la teoría del consentimiento}

Aunque ya existe cierto consenso entre los exégetas de que la teoría del derecho de resistencia de Locke no es puramente original, sino que tiene múltiples antecedentes teóricos, no por esto deja de ser atractiva. Al contrario, las sutilezas y matices de la misma, así como su renovado interés en los estudios lockeanos han hecho que ella no pierda vigencia. Más precisamente, para su adecuada comprensión, debemos tener en cuenta que Locke opera una distinción entre el poder que constituye el gobierno, el poder constituyente, y el "órgano" que detenta el ejercicio del poder político propiamente dicho. Para ahondar en la distinción resultará conveniente explicitar la definición lockeana del poder político:

Entiendo que el poder político consiste en el derecho de establecer leyes con la pena de muerte y, consecuentemente con todas las penas menores, para la regulación y preservación de la propiedad, y en el uso de la fuerza de la comunidad, al ejecutar esas leyes, y en la defensa del Estado [Common-wealth] frente a agresión extranjera, todo esto sólo en aras del bien público ${ }^{1}$.

Podríamos sugerir que en esa definición ya se encuentran expresados casi todos los elementos que competen a la distinción antes mencionada. Se observa, en primer lugar que se apela al "derecho de establecer leyes", en segundo lugar al "uso de la fuerza de la comunidad al ejectuar esas leyes", en tercer lugar a la "defensa del Estado frente a la agresión extranjera" y, finalmente, la supeditación de todos estos elementos al "bien público". Uno podría asimilar cada uno de los tres primeros elementos al poder Legislativo, el poder Ejecutivo y el poder Federativo del Estado, en cuya descripción

\footnotetext{
${ }^{1}$ J. LOCKE, Two Treatises of Government, Cambridge, Cambridge University Press, 1988, 286, II \3. Todas las traducciones de Locke y los intérpretes son propias.
} 
abunda el capítulo XII de los Two Treatises. Más aún, aunque Locke también aclare en el $\int$ 150 que en todos los casos en los que subsistan el gobierno y sus tres poderes, el Legislativo siempre será el "poder supremo", todos ellos tienen su origen y dependen, en definitiva, como poderes fiduciarios ${ }^{2}$, del "trust" de la "comunidad" cuya fuerza utilizan y cuya propiedad defienden en aras del "bien público".

A su vez, los orígenes de dicha comunidad se encuentran descritos con precisión en el capítulo VII de la misma obra:

Siendo los hombres, como se ha dicho, todos libres, iguales e independientes por naturaleza, ninguno puede ser puesto fuera de este estado y sometido al poder político de otro sin su propio consentimiento. La única manera por la que uno se despoja de su libertad natural y se incorpora a la sociedad civil [puts on the bonds of Civil Society] es por medio de un acuerdo con los otros hombres en juntarse y unirse en una comunidad, para su vida confortable, segura y pacífica entre sí en el seguro disfrute de sus propiedades $(\ldots)$ Cuando cualquier número de hombres ha consentido formar una comunidad o un gobierno, entonces se encuentran incorporados y constituyen un cuerpo político, en el que la mayoría tiene el derecho de actuar y decidir por el resto ${ }^{3}$.

En dicho párrafo se observa que el origen de la comunidad política depende de la salida del estado de naturaleza descrito en el II, $\$ 4$ de los Two Treatises, en los que cada persona tiene juicio final sobre la ley de naturaleza. Tal juicio es reemplazado, en la sociedad civil, por el de la mayoría, dado que de otra manera el cuerpo político quedaría paralizado y no tendría moción. $\mathrm{Y}$, precisamente, el cuerpo político debe tener la fuerza suficiente para realizar sus fines, descritos en II, $\$ 123: 《(.$.$) la mutua preservación, de sus vidas, libertades$ y bienes, que yo llamo por el nombre general, propiedad». Esto tiene como consecuencia que todo aquél que forme parte de la comunidad política también ponga sus propiedades bajo jurisdicción de la misma:

\footnotetext{
${ }^{2}$ En II, \$149 Locke explicita que la relación del Legislativo con la comunidad es la de un poder fiduciario y que el poder que éste regula y el ejecutivo controla es sólo un "trust" condicionado a los fines que dan origen a la comunidad política.

${ }^{3} \mathrm{~J}$. LOCKE, Two Treatises, 330-331, II \$95. Las bastardillas son originales del texto lockeano.
} 
[T]odo hombre, cuando él, en principio, se incorpora a cualquier Estado, por su unión con él, anexa también y somete a la comunidad aquellas posesiones que tiene o que llegará a adquirir, que no pertenezcan ya a algún otro gobierno ${ }^{4}$.

En este contexto, sin embargo, todavía es posible que la distinción entre el poder constituyente y el poder político quede desdibujada por los sucesivos capítulos en los que Locke desarrolla su modelo de institucional. En los capítulos IX al XIV de los Two Treatises, es decir, aquéllos inmediatamente posteriores a la explicitación de las condiciones y los motivos que dan lugar a la formación del Estado, el autor desarrolla las relaciones internas de los distintos poderes de poder político. Sólo en el II, \$133 hay un breve indicio de que el Estado lockeano debe distinguirse de cualquier forma de gobierno y conceptualizarse, antes bien, como una "sociedad de hombres".

La distinción, sin embargo, se hace patente en el capítulo XIX en el que, no casualmente, se expone la doctrina del derecho de resistencia. Si bien el tema que compete a ese capítulo es la disolución, en particular, del gobierno, lo primero que se postula, ya en el $\$ 211$, es que la única causa por la que la sociedad civil se disuelve es por la conquista exterior, circunstancia en la cual el gobierno de dicha sociedad tampoco puede subsistir. En efecto, en los siguientes apartados Locke desarrolla las distintas causas por las que puede disolverse el gobierno, que se agrupan en dos posibilidades. Según la primera, el gobierno se disuelve cuando el poder legislativo es objeto de alteración. Esta alteración, a su vez, se manifiesta de distintas maneras: (i) cuando el príncipe erige su voluntad arbitraria sobre las leyes (II, \$214), (ii) cuando el príncipe no permite reunirse al Legislativo (II, \$215), (iii) cuando los electores o procedimientos de elección del Legislativo son modificados (II, \$216), (iv) cuando el príncipe o la legislatura entregan al pueblo al dominio extranjero (II, \$217) y finalmente (v) cuando quien detenta el poder ejecutivo abandona el cargo, lo cual hace inaplicables a las leyes (II, \$219).

La segunda posibilidad de disolución del gobierno es aquélla según la cual el príncipe o el poder Legislativo actúan en contra del "trust" que le ha concedido al pueblo. La fórmula de dicha causa de disolución del gobierno en $₫ 221^{5}$ es casualmente semejante a, aunque supone la violación de cada uno de los términos de $\$ 134$ en los que

\footnotetext{
${ }^{4} \mathrm{~J}$. LOCKE, Two Treatises, 348, II $\$ 120$.

${ }_{5}$ Para el caso del poder legislativo. La misma fórmula, en caso de que sea el poder ejecutivo el que viole el “trust" se encuentra en $\$ 222$.
} 
se asocian los fines por los cuales se entra en sociedad a la constitución del poder legislativo:

Primero, el legislativo actúa contra el trust depositado en él, cuando intenta invadir la propiedad del súbdito y hacerse a sí mismo, o a parte de la comunidad, amos o disponer arbitrariamente de las vidas, libertades o fortunas del pueblo ${ }^{6}$.

En todos estos casos, pero más aún en aquéllos en los que se imputa la violación del "trust", podría pensarse que un solo individuo, presumiblemente el damnificado, está facultado para resistir al gobierno. Sin embargo, Locke nos ofrece razones tanto pragmáticas como teóricas para rechazar esta hipótesis. Encontramos la razón pragmática en II \$208, donde se afirma que aunque el individuo tenga derecho a defenderse, ni él ni alguna parte, presumiblemente minoritaria, del pueblo se involucrarán en conflictos en los que serán derrotados ${ }^{7}$. Por otra parte, las razones teóricas por las que la resistencia lockeana adquiere un carácter fundamentalmente colectivo ${ }^{8}$ apelan a dos potestades que sólo puede ejercer el pueblo de manera colectiva. Una de ellas hace al pueblo entero el juez último respecto a los conflictos que se susciten entre quienes ejercen el poder político, ya sea en la forma del Ejecutivo o del Legislativo y parte del pueblo:

(...) ¿Quién será el juez acerca de si el príncipe o el legislativo actúan contra su "trust"?

(...) A esto respondo, el pueblo será juez (... $)^{9}$

Y en el mismo sentido:

\footnotetext{
${ }^{6} \mathrm{~J}$. LOCKE, Two Treatises, 412, II $\$ 221$.

7 Asimismo, a lo largo de $\$ 223$ y $\$ 225$ Locke asegura que el pueblo tiende a conservar sus formas y costumbres antes que a cambiarlas y que incluso soportará varios errores de la administración sin resistirse. En II $\$ 230$ se indica que sólo atentados manifiestos contra el pueblo lo llevan a sublevarse y no tumultos aislados, como podría ser la damnificación de un solo individuo.

${ }^{8}$ En D.A. Fernández Peychaux, "La disolución de la personal majesty: el derecho de resistencia comunitario lockeano", Themata. Revista de Filosofía, No 45 (2012), 131-132 y J.S. CHUMBITA, "La configuración del pueblo en la resistencia. Un abordaje contemporáneo sobre la obra de John Locke", Revista SAAP, Vol. 8, $\mathrm{N}^{\circ} 1$ (2014) 181-182 se abunda en la forma colectiva que adopta la resistencia lockeana.

9 J. LOCKE, Two Treatises, 427, II $\$ 240$.
} 
Si surge una controversia entre el príncipe y parte del pueblo (...) yo considero que el árbitro apropiado en ese caso debería ser la totalidad del pueblo $(. . .)^{10}$

La otra potestad claramente colectiva del pueblo es la de, toda vez que se disuelva el gobierno, erigir un nuevo poder Legislativo:

Cuando suceda que el Legislativo transgreda esta regla fundamental de la sociedad (...) por esta violación del "trust" ellos relegar su poder [forfeit the Power], que el pueblo había puesto en sus manos para fines contrarios, y éste vuelve al pueblo, que tiene derecho de recuperar su libertad original y, establecer un nuevo legislativo (así como ellos lo consideren apropiado) que provea su seguridad $(. . .)^{11}$.

Aquella "libertad" que recupera el pueblo, nuevamente, no debe ser comprendida de manera individual, como un retorno al Estado de naturaleza de cada uno ${ }^{12}$. Al contrario, constituye un lapso en el que el pueblo ejercita, como sociedad civil bajo regla de la mayoría, su voluntad y constituye nuevamente el Legislativo para, valga la redundancia, cederle a éste poder la manifestación de su voluntad. La tesis del retorno de la soberanía al pueblo, antes que a cada individuo, concluye los Two Treatises:

Para concluir, el poder que cada individuo entregó a la sociedad, cuando entró en ella, no puede volver nuevamente al individuo, en tanto la sociedad se mantenga, sino que siempre permanecerá en la comunidad; porque sin este poder no puede haber comunidad, ni estado, lo que es contrario al acuerdo original $(\ldots)^{13}$.

Entonces, la estrategia conceptual de Locke para poder postular una teoría de la resistencia sin comprometerse, también, con consecuencias anárquicas consiste en separar el poder constituyente del poder político. Toda vez que se disuelva el gobierno, caerán con él como rebeldes los funcionarios que ejercen el poder político, pero éste último volverá a las manos del pueblo en tanto poder constituyente. Ya adelantamos que

\footnotetext{
${ }^{10} \mathrm{~J}$. LOCKE, Two Treatises, 427, II $\$ 242$.

${ }^{11} \mathrm{~J}$. LOCKE, Two Treatises, 412-413, II $§ 222$.

${ }_{12}$ Aunque sí representa un regreso al Estado de naturaleza entre el pueblo y los funcionarios que atentaron contra el trust, en condición de rebeldes.

${ }^{13} \mathrm{~J}$. LOCKE, Two Treatises, 427-428, II \$243.
} 
la afirmación de un derecho de resistencia no es nueva para la teoría política. Tampoco lo es, en particular, esta concepción dual del poder que puede ser rastreada por lo menos al Politica Sacra e Civilis de George Lawson"14, en donde se distingue entre la "real majesty" del pueblo y la "personal majesty" del rey y el parlamento.

Lo que nos interesa y llama al cuestionamiento es, sin embargo, uno de los elementos de este último punto. Si la resistencia retrotrae el poder político al pueblo, como poder constituyente, no resultará insignificante ni trivial determinar las condiciones por las cuales se puede pertenecer a ese cuerpo político. De acuerdo a los criterios de membrecía y pertenencia, la teoría lockeana podría tomar una forma ampliamente democrática o, por el contrario, resultar en una oligarquía notablemente restringida. Así, la resistencia contra el tirano podría estar protagonizada por una concepción amplia del pueblo que involucra a todos los habitantes de, por ejemplo, Inglaterra o bien reducirse a intrigas palaciegas y manipulaciones políticas por parte de los nobles.

$\mathrm{Al}$ menos en una primera lectura, Locke es claro respecto al criterio de membrecía al Estado. Éste consiste en nada menos que el consentimiento expreso del individuo en cuestión:

Siendo todo hombre, como se ha mostrado, naturalmente libre y no habiendo nada que pueda ponerlo bajo sometimiento de un poder terrenal sino si propio consentimiento; debemos considerar qué se entenderá como una declaración suficiente de consentimiento, que lo haga súbdito a las leyes de algún gobierno (...) Nadie duda de que sólo el consentimiento expreso, de cualquier hombre, entrenado en sociedad lo hace a éste un miembro perfecto de aquélla sociedad, un súbdito de ese gobierno $(\ldots)^{15}$.

Si una persona que presenta su consentimiento expreso a entrar en sociedad con otras, forma parte de aquél pueblo que puede ejercer el derecho de resistencia cuando el poder político no respeta el "trust", parece difícil decir lo mismo de aquéllos que sólo han consentido tácitamente a obedecer las leyes del Estado. En efecto, en paralelo al

${ }^{14}$ Un análisis más exhaustivo de la influencia del argumento lawsoniano en Locke puede encontrarse en D.A. FERnÁndeZ Peychaux, "La disolución de la personal majesty", 126. C. CONDREN, "Resistance and Sovereignty in Lawson's Politica: An Examination of a Part of Professor Franklin, His Chimera" The Historical Journal, Vol. 24, $\mathrm{N}^{\circ} 3$ (1981), 673 revisa la teoría lawsoniana y pone en duda la interpretación de J. FrankLIN, John Locke and the Theory of Sovereignty, Cambridge, Cambridge University Press, 1978, que atribuye consecuencias "democratizantes" a su argumento.

15 J. LOCKE, Two Treatises, 349, II \$122. 
consentimiento expreso, Locke considera que el consentimiento tácito de aquéllos que usufructúan propiedades en el territorio de un gobierno o transitan por él:

\begin{abstract}
Someterse a las leyes de cualquier país, vivir tranquilamente y disfrutar de los privilegios y protección bajo ellas no hace a un hombre miembro de esa sociedad. Esto es sólo la protección local y deber de homenaje [Homage] que se debe a todos aquéllos que, no estando en un estado de guerra, llegan a los territorios que pertenecen a un gobierno, a todas las partes en las que se extiende la fuerza de sus leyes ${ }^{16}$.
\end{abstract}

Un análisis adecuado de la distinción entre estos dos tipos de consentimiento puede ser útil para acotar la clase de individuos capaces de obtener la membrecía al Estado lockeano. Si, por ejemplo, hay una tipo de personas que sólo puede prestar consentimiento tácito, entonces sabremos con certeza que no pertenecen al pueblo que puede ejercer su derecho de resistencia. En la siguiente sección reseñaremos dos enfoques estrictamente normativos de la noción de consentimiento. Mientras que señalaremos los puntos altos de las mismas, intentaremos mostrar que no aportan elementos conclusivos para el análisis de la concepción del pueblo lockeano a partir de la distinción entre los dos tipos de consentimiento.

\title{
III. Los límites normativos del consentimiento lockeano
}

En un ya clásico trabajo titulado "Obligation and Consent" Pitkin ofreció un innovador análisis de la teoría lockeana del consentimiento. De acuerdo a su lectura, normalmente se ha creído que la premisa fundamental del argumento de Locke hace que la obligación política de obedecer dependa de algún tipo de acto de consentimiento. En principio, éste tipo de acto es el consentimiento expreso que Locke desarrolla en el \$119 de los Two Treatises. Precisamente, Pitkin encuentra que el acto del consentimiento expreso es una forma de promesa. De acuerdo a Locke, toda persona tiene la obligación de cumplir con aquello que haya prometido:

\footnotetext{
${ }^{16} \mathrm{~J}$. LOCKE, Two Treatises, 349, II \122.
} 
Es verdad que respecto a cualquier compromiso o promesa que uno ha hecho, se encuentra bajo la obligación de cumplirla ${ }^{17}$.

Sin embargo, en el mismo $\$ 119$, Locke establece que no sólo ese tipo de consentimiento, expreso, sino también el consentimiento tácito dispara la obligación de obediencia a un gobierno. Este tipo de consentimiento consiste en la posesión o el usufructo de propiedad en el territorio de un gobierno, el tránsito por su territorio e incluso «(...) en efecto, se extiende al punto de que obliga tan sólo al encontrarse uno en cualquiera de los territorios de ese gobierno» ${ }^{18}$. Pitkin sugiere que la adición del consentimiento tácito como generador de una obligación de obedecer responde al problema de que no todos las personas han, de hecho, consentido expresamente obedecer a un gobierno determinado. Ahora bien, para la autora la extensión del consentimiento expreso en consentimiento tácito conlleva un doble problema fundamental para la teoría lockeana. Por una parte la noción de consentimiento pierde sentido dado que parece alcanzar prácticamente cualquier acción que uno realice y, así, «todos están automáticamente obligados [a obedecer]» ${ }^{19}$. Por la otra, como corolario de esta extensión toda persona que se encuentre incluso en el territorio de un gobierno "tiránico" se encuentra obligado a obedecerlo dadas las muy amplias condiciones del consentimiento tácito, sin posibilidad de resistencia más allá del exilio.

Pitkin sugiere que dicha lectura no puede ser una exposición correcta de la teoría lockeana, si es que ésta debe hacer consistente la distinción entre ambos tipos de consentimiento con el derecho de resistencia y una distinción adecuada entre autoridad legítima y mera coerción. La solución que ella ofrece consiste en leer en el texto de Locke una teoría de la obligación política basada en las "características del gobierno". De acuerdo a aquél. El siguiente pasaje resume la posición de la autora:

\footnotetext{
${ }^{17} \mathrm{~J}$. LOCKE, Two Treatises, 346, II \$116.

${ }^{18} \mathrm{~J}$. LOCKE, Two Treatises, 349, II \$119.

${ }^{19}$ H. PITKIn, "Obligation and Consent I", The American Political Science Review, Vol. 59, Nº 4 (1965), 995.

El agregado entre corchetes es nuestro.
} 
En tanto las acciones de un gobierno estén dentro de los límites de lo que bubiera provisto un contrato hipotético semejante o tendría que haber provisto, aquéllos viviendo en dicho territorio deben obedecer ${ }^{20}$.

A. J. Simmons también ha revisado la teoría lockeana del consentimiento en varios capítulos de su libro Moral Principles and Political Obligation. En dicho texto, el comentarista lleva a cabo una tarea crítica, por una parte, sobre el argumento del consentimiento expreso de Locke. Por otra, tampoco considera que la reconstrucción de Pitkin sea aceptable y, finalmente, también encuentra equivocado el argumento lockeano del consentimiento tácito. Simmons parece convencido de que sólo el consentimiento expreso funda una obligación de obedecer, pero que la falta de dicho consentimiento expreso por parte de las personas hace que sea imposible afirmar que existe una obligación de obedecer a los gobiernos por parte de sus ciudadanos ${ }^{21}$.

La crítica de Simmons al argumento de Pitkin comienza, por su parte, con una reconstrucción del mismo. Podemos sintetizarlo con la siguiente versión abreviada del mismo: (i) La residencia siempre es un acto de consentimiento (tácito), (ii) Debemos obedecer a los gobiernos "buenos" pero no a los gobiernos "tiránicos" o "despóticos" y (iii) El consentimiento funda la obligación política. Según Simmons, Pitkin compatibiliza (i) y (ii) al desprenderse de la premisa (iii) y enfocar su lectura en la teoría de las "características del gobierno". Simmons considera que Pitkin se compromete con una lectura forzada del texto lockeano porque "parece ignorar la posibilidad de que el consentimiento sea sólo una condición necesaria, antes que una suficiente, de la generación de la obligación política" 22 . Este autor ilustra la distinción con el siguiente ejemplo: dada una promesa de cometer un crimen perverso y dada otra promesa de donar la mitad de mis ingresos a un amigo necesitado, nuestras intuiciones nos indican que mientras que ambas son promesas "reales", sólo la segunda nos obliga. De acuerdo a la lectura de Pitkin, mientras que la primera promesa jamás nos obligaría, la segunda nos obligaría independientemente de ser una promesa, por el sólo hecho de su contenido moralmente encomiable. Simmons, al contrario, considera que la obligación se deriva del

\footnotetext{
${ }^{20}$ H. PITKIN, "Obligation and Consent I", 996.

${ }^{21}$ Cfr. A. J. Simmons, Moral Principles and Political Obligation, Princeton (NJ), Princeton University Press, $1979,73$.

22 A. J. Simmons, Moral Principles and Political Obligation, 86.
} 
acto de prometer mientras que la cualidad moralmente repudiable de la primera promesa meramente impide que ésta me obligue.

En este sentido, Simmons considera que la posición de Locke es que nuestro consentimiento sólo nos obliga cuando se da a "buenos" gobiernos, pero que es el consentimiento lo que funda la obligación. De esta manera, se puede evitar la conclusión problemática de que la residencia en el territorio de un gobierno tiránico genera la obligación de obedecerlo. Si el consentimiento es sólo una condición necesaria de la obligación política, es perfectamente posible incluir la premisa adicional de que dicho consentimiento debe ser a un gobierno "bueno" para que se funde una obligación válida. Así, ni la residencia en el territorio de un gobierno tiránico ni la residencia en el territorio de un gobierno "bueno" generan obligación alguna.

Resulta patente, sin embargo, que la propuesta de Simmons parece contradecir parte del texto lockeano, específicamente en lo que hace al alcance del consenso tácito como generador de obligación política en el \$119. En efecto, del párrafo anterior parece concluirse que en la teoría de Locke no es posible ninguna clase de obligación política sino aquélla que se deriva del consentimiento expreso, en oposición al repetido uso que Locke hace del dispositivo del consentimiento tácito. Para Simmons ninguno de los actos que para Locke ejemplifican consentimiento tácito, ya sea tener una propiedad, como usufructuarla, transitar por las rutas, o residir en un país, son verdaderos actos de consentimiento. Al contrario, este comentador sugiere que «Locke confusamente etiqueta el disfrute de los beneficios del gobierno como un caso especial de un acto consensual el consentimiento "tácito". Pero hemos visto que mientras que los "disfrutes" de Locke pueden "implicar consentimiento" y por ello podría tener "algo que ver" con el consentimiento personal, no son "signos de consentimiento" 23 . Mientras que los actos que son "signos de consentimiento" serían genuinos actos consensuales que expresan la intención de un sujeto de consentir, aquéllos que "implican consentimiento" son actos que obligan moralmente a un sujeto como si hubiera consentido, pero a partir de otro tipo de principios. En consecuencia, según el comentarista, los tipos de actos descritos en el \$119, en la caracterización del consentimiento tácito generarían obligaciones de obediencia pero no a partir de un principio de consentimiento, sino a partir de consideraciones de equidad (fairness) o gratitud, que Locke no considera.

${ }^{23}$ A. J. SIMMONS, Moral Principles and Political Obligation, 91. 
Es difícil cuestionar que ambas lecturas del problema de la obediencia política son muy ilustrativas de los límites de los argumentos basados en el consentimiento. En ese sentido tanto Pitkin como Simmons desarman las premisas del argumento y sugieren algunos refinamientos para hacerlo más sólido, donde sea posible, así como señalan aquéllas perspectivas donde el argumento no puede funcionar. Sin embargo, consideramos que ambos se centran solamente en establecer si el texto lockeano, a partir del argumento del consentimiento, logra asegurar un deber de obediencia a las leyes del Estado y las razones por las que dicho deber tendría validez. El análisis no se detiene en observar también qué consecuencias tiene la distinción entre el consentimiento expreso y el consentimiento tácito respecto a otro tipo de deberes de los individuos respecto al Estado y el poder político. En la siguiente sección desarrollaremos estos elementos a los que, creemos, los análisis desde perspectivas como las de Pitkin y Simmons no llegan.

\section{Consentimiento expreso y deberes en el pueblo lockeano}

Como se mencionó previamente, para Locke el consentimiento tácito sólo trae aparejado el deber de obedecer las leyes de un gobierno. El consentimiento expreso, no sólo trae aparejado dicho deber. También incluye otros deberes que pueden servir de indicio para determinar quiénes pueden consentir expresamente, en función de quiénes pueden llegar a cumplir con esos deberes. El primero y principal de estos deberes, formulado en II \$120, consiste en el de anexar a la jurisdicción del Estado al que se consiente expresamente, todas las propiedades que un individuo tiene y tendrá.

Es conocida la polisemia que adquiere la palabra "propiedad" a lo largo de los Two Treatises, magistralmente exhibida en el siguiente pasaje ${ }^{24}$ : «sus vidas, libertades y bienes, que yo llamo por el nombre general, propiedad» ${ }^{25}$. En consecuencia, todo aquél individuo capaz de ser miembro de la sociedad debe tener propiedad para anexar al Estado. En una interpretación débil de este requisito, todo individuo poseería propiedad para anexar, en tanto posee su propio cuerpo. En una interpretación restringida, sin embargo, la propiedad relevante en este caso es la tierra. Más adelante veremos que se

\footnotetext{
24 Aunque también se manifiesta en muchos otros, por ejemplo II \$173. La importancia de esta ambigüedad para la teoría política lockeana también es señalada por J. S. CHUMBITA, "Apropiación privada de la tierra y derechos políticos en la obra de John Locke", Ingenium. Revista de historia del pensamiento moderno, $\mathrm{N}^{\circ} 7$ (2013), 193.

25 J. LOCKE, Two Treatises, 350, II \$123.
} 
han ofrecido muchas interpretaciones que encuentran en el requisito de la posesión de tierra la piedra de toque para una restricción del pueblo lockeano a los terratenientes.

Antes de adentrarnos el requisito de posesión de tierra, sin embargo, debemos repasar en un segundo deber positivo lockeano para los miembros de la sociedad. Nos referimos al de pagar impuestos para mantener al Estado:

Es verdad que los gobiernos no pueden ser mantenidos sin un gran expendio, y es apropiado que todo aquél que disfruta de su protección, deba pagar con su patrimonio su proporción por su mantenimiento ${ }^{26}$.

Una primera lectura de este deber parece suscitar los mismos interrogantes que el de la anexión de propiedad al Estado. En principio, para que cualquier individuo pueda contribuir a los impuestos, si es que goza de alguna protección, debe tener bienes (en dinero u otras especies) con que pagarlos. Ahora bien, el deber de pagar impuestos no se agota en la problemática de si un individuo es capaz de pagarlos o no, sino que además condiciona la representación política parlamentaria. Analizando el posible contexto del cambio del Legislativo por parte del Ejecutivo, Locke presenta la tesis según la cual la representación parlamentaria debe ser proporcional a la contribución impositiva de cada “sector" del Pueblo:

Si el poder ejecutivo, que tiene la potestad de convocar al legislativo, observa la proporción verdadera antes que la costumbre de representación y regula, no por viejas tradiciones, sino por la verdadera razón que el número de miembros, en todos los lugares, que tiene derecho a ser distintamente representados, a lo que ninguna parte del pueblo puede pretender, se establece en proporción a la asistencia que cada uno otorga al Estado $^{27}$.

Así, la tesis de la "verdadera proporción" parece hacer equivaler el grado de contribución impositiva al «a la representación justa e igualtaria» que Locke menciona más abajo en el mismo \158. El parlamento lockeano, entonces, parece quedar lejos de una forma de 
"democracia perfecta" 28 y asumir, en cambio, formas estamentales muy semejantes a las del gobierno inglés de su época, descrito para un argumento hipotético en \$213.

Señalados ya los dos particulares que parece implicar la membrecía a la sociedad lockeana, el de anexar la propiedad y el de mantener el Estado con impuestos, podemos ponderar los distintos argumentos que, a partir de ellos, se han ofrecido para ampliar o restringir el concepto de pueblo. En este sentido, el primero de los argumentos que vale la pena mencionar es el de James Tully en $A$ Discourse on Property. Este comentador se centra en una interpretación débil del requisito de propiedad y considera que a partir de dicho requisito Locke deriva amplios derechos políticos de representación: «Al demostrar que todo hombre tiene propiedad sobre su vida, libertad, persona, acción y algunas posesiones, Locke extiende la franquicia [de votación] a todo hombre adulto. Él no explicita el criterio en los Two Treatises; simplemente lo asume como la base en su discusión de varios tipos de representación (...)»²9 (Tully 1980:173, los corchetes son nuestros). El argumento de Tully, sin embargo, no puede ser aceptado sencillamente. En primer lugar, como hemos señalado, hay una distinción entre la membrecía al pueblo, el poder constituyente, y el poder político. Una interpretación débil del requisito de propiedad quizás pueda afirmar una amplia membrecía popular, en tanto toda persona tiene algo que anexar al Estado. La misma interpretación, empero, es insuficiente para afirmar derechos políticos democráticos (para todo hombre adulto). Como se ha señalado previamente, correlativa al deber de pagar impuestos se configura la representación política parlamentaria que, nuevamente, depende de la proporción de lo contribuido. Que toda persona tenga algo con lo que pagar algún impuesto no significa que pueda pagar lo mismo y, en ese sentido, que tenga igual derecho a ser representada.

Neil Wood, en su libro John Locke and agrarian capitalism, formula este tipo de crítica a la propuesta de Tully. De acuerdo a este autor, no hay evidencia textual que permita afirmar el salto conceptual desde una interpretación débil del requisito de propiedad hacia una ampliación democrática de los derechos políticos ${ }^{30}$. A su vez, repara en que para la época de la revolución inglesa la franquicia de representación

\footnotetext{
${ }^{28}$ Así es como Locke denomina, en II \$132, a una forma de gobierno en el que la mayoría dicta las leyes de la comunidad.

${ }^{29} \mathrm{~J}$. Tully, A Discourse on Property. John Locke and his adversaries, Cambridge, Cambridge University Press, 1980, 173. Los corchetes son nuestros.

${ }^{30}$ Cfr. N.WoOD, John Locke and agrarian capitalism, Los Angeles, University of California Press, 1984, 84.
} 
definitivamente se había ampliado y que, en el contexto de la crisis de exclusión, el partido Whig podría haber intentado una ampliación de la misma para frustrar el poder de la corona.

Esta última es la perspectiva que han adoptado Richard Ashcraft y Julian Franklin en Revolutionary Politics and Locke's Two Treatises of Government y John Locke and the Theory of Sovereignty, respectivamente. De acuerdo al primero, el capítulo XIX de los Two Treatises tenía por sentar las bases de un movimiento político radical en colaboración con Ashley Cooper, el primer conde de Shafterbuty ${ }^{31}$. El autor precisa, además, que dicho movimiento sostenía ideales igualitarios y apelaba a argumentos "leveller". Por su parte, Franklin sugiere que Locke adoptó el argumento lawsoniano de la distinción entre la "personal majesty" del rey y la "real majesty" del pueblo para que, toda vez que fallara la primera, el poder se retrotrajera al segundo ${ }^{32}$.

Aunque fuera, en principio, posible atribuirle semejantes intenciones revolucionarias a Locke y encontrar algunos pasajes a favor de la tesis que hace del autor un revolucionario radical, no es posible dejar de mencionar el convincente argumento de David Wootton en el artículo "John Locke and Richard Ashcraft's Revolutionary Politics". Allí el autor ofrece abundante evidencia textual en contra del radicalismo propuesto por Ashcraft y Franklin. La primera pieza documental que merece mencionarse es The Foundamental Constitutions of Carolina, en la que Locke y Shaftesbury plasman el diseño institucional de la colonia. En contra de todo posible radicalismo, se establece la posesión de cantidades específicas de acres como condición para ejercitar derechos políticos ${ }^{33}$. Una segunda pieza documental de importancia señalada por Wootton es la An Essay on the Poor law ${ }^{34}$, en la que, según éste, se manifiesta la dureza de Locke para tratar la cuestión de la pobreza. Así procede Wootton al tratamiento de varios documentos más, entre ellos por ejemplos Venditio o Labor, para concluir parcialmente:

El resultado, me parece, es que uno puede hablar acerca de conflictos y tensiones en el pensamiento de Locke, pero no está capacitado para retratarlo como un radical

\footnotetext{
31 Cfr. R. ASHCRAFT Revolutionary Politics and Locke's Two Treatises of Government, Princeton (NJ), Princeton University Press, 1986, 309.

${ }^{32}$ Cfr. J. FRANKLIN, John Locke and the Theory of Sovereignty, 88 y ss.

${ }^{33} \mathrm{El}$ documento se encuentra en J. LoCKE, The Works of John Locke, London, Rivington, Vol. 9, 1685.

${ }^{34} \mathrm{El}$ documento se encuentra editado en J. LOCKE, Political Essays, Cambridge, Cambridge University Press, 2004.
} 
consistente. Existen textos radicales pero no hay evidencia de que hubiera un autor radical $^{35}$.

El análisis de Wootton, sin embargo, tampoco concluye con esos documentos. En efecto, su punto más fuerte en contra de las tesis de Ashcraft y Franklin se encuentra al revisar la posición de Locke sobre la constitución inglesa. El requisito para poder sugerir la tesis de Locke como un pensador radical queda así descrito:

Para que Ashcraft tenga razón, Locke necesita ofrecer, en el Segundo tratado, una definición de pueblo que sea inclusiva, antes que exclusiva, y los Whig radicales necesitan haber creído en una extensión, no en una restricción de la franquicia ${ }^{36}$.

Como se adelantó, sin embargo, Wootton presenta evidencia en contra de esa posibilidad. En primer lugar, hace referencia a una carta de Locke a Edward Clarke en 1689 en la que sucesivas veces manifiesta su acuerdo con la conformidad de Guillermo, príncipe de Orange, respecto a que el parlamento de la convención, limitado como era en su representación, fuera, valga la redundancia, representativo de toda la nación. Esto se confirma en un pequeño texto en el que Locke exhorta a la población inglesa a declarar su adhesión al ahora rey Guillermo $\mathrm{III}^{37}$ para mantener la estabilidad pública:

(...) but 'tis impossible to be expected that those should support any constitution who will not so much declare for it and own the right of the prince they are to obey. This is that which all constitutions which outlasted their birth have constantly taken care of in the first place; the right of their governors and the declaration and owning of that right never left loose and uncertain ${ }^{38}$.

${ }^{35}$ D. WoOtTON, "John Locke and Richard Ashcraft's Revolutionary Politics”, Political Studies, Vol. 40 (1992), 91.

${ }^{36}$ D. WoOTTON, “John Locke and Richard Ashcraft's Revolutionary Politics”, 93-94.

${ }^{37} \mathrm{Al}$ que, podríamos pensar, Locke se refiere como "those whose business is to take care of the public will secure that as it ought". Cfr. J. LOCKE, "On Allegiance and the Revolution" en J. LOCKE: Political Essays, Cambridge, Cambridge University Press, 1997, 307.

${ }^{38}$ J. LOCKE, "On Allegiance and the Revolution", 312. 
La lectura de Ashcraft ${ }^{39}$, al contrario que la ya tantas veces criticada posición de Macpherson ${ }^{40}$, parece tomar una versión débil del requisito de propiedad para ampliar las bases populares lockeanas. Sin embargo, Wootton indica, esto choca con la aceptación del parlamento de la convención de 1689 por parte de Locke, en la que no había representación igualitaria de todos los sectores del pueblo. Uno podría intentar rescatar la tesis de Ashcraft con la alternativa conceptual antes mencionada de Franklin, que hace uso de la distinción de corte lawsoniano entre el poder político (“personal majesty”) y el poder constituyente del pueblo ("real majesty"). Según ésta, recordemos, cuando se disuelve el poder político, éste retorna al pueblo. Franklin considera que esta combinación de la distinción lawsoniana tiene para Locke consecuencias "leveller", en tanto entiende el pueblo de manera amplia. Pero tanto Wootton y Condren ${ }^{41}$ indican que el mismo Lawson no suscribía una noción amplia de pueblo sino que éste se limitaba a terratenientes con propiedades cuyo valor superaba un mínimo establecido. Así, según Wootton, el argumento lockeano contiene elementos de Lawson, pero sin las consecuencias radicales que Ashcraft y Franklin le agregan. Ante la disolución del gobierno, ni se vuelve al estado de naturaleza ni se retrotrae necesariamente el poder a todas las personas, sino sólo a los "miembros existentes de la comunidad política" ${ }^{42}$. Y nuevamente, si se adopta una interpretación restringida basada en tierra del requisito de anexión de propiedad ${ }^{43}$, la comunidad política será muy restringida.

\footnotetext{
39 Debemos notar que Wootton, entre otros, ha llamado la atención sobre una aparente contradicción en el texto Lockeano. En II \$117, Locke parece hacer necesario ser miembro de la comunidad, en el caso de la herencia, para usufructuar la tierra en el territorio de un gobierno mientras que, como se mencionó antes, en el II $\$ 119$ se establece que el usufructúo de la tierra sólo equivale a consentir tácitamente obedecer las leyes de un gobierno.

40 Macpherson argumentaba que Locke limitaba la membrecía a la sociedad a los grandes terratenientes, mientras que, por el recurso del consentimiento tácito, obligaba a los trabajadores a obedecer. La obra de Locke se configuraba así como una de las primeras defensas teóricas del capitalismo y el "individualismo posesivo". Desde su publicación, ha recibido numerosas críticas no sólo por extrapolar categorías del siglo XIX en el siglo XVII, sino por no lograr articular una lectura consistente. Cfr. C. B. MARCPHERSOn, The Political Theory of Possesive Individualism, Oxford, Oxford University Press, 1962, y para algunas críticas cfr. D. WOOTTON, "John Locke and Richard Ashcraft's Revolutionary Politics", 94 y J. DunN, "Consent in the Political Theory of John Locke", The Historical Journal, Vol. X, N 2 (1967) 165166.

${ }^{41}$ Cfr. D. WootTon, “John Locke and Richard Ashcraft's Revolutionary Politics”, 95 y C. CONDRen, "Resistance and Sovereignty in Lawson's Politica", 680.

${ }^{42}$ D. WOOTTON, "John Locke and Richard Ashcraft's Revolutionary Politics”, 96.

${ }^{43}$ Como lo sugiere el mismo comentador. Cfr. D. WoOTTON, "John Locke and Richard Ashcraft's Revolutionary Politics”, 96.
} 
Un último texto sobre el que queremos reparar introduce, sin embargo, una posición algo paradójica sobre lo previamente desarrollado. En Some Considerations of The Consequences of the Lowering of Interest, and Raising the Value of Money, Locke parece atribuir a los trabajadores (labourers) tanto una incapacidad para lo político como una capacidad específica para la resistencia:

El salario del trabajador, siendo pocas veces mayor que una mera subsistencia, nunca permite a este cuerpo de hombres tener el tiempo o la oportunidad para elevar sus pensamiento sobre ello, o para unirse y pelear contra los ricos por lo suyo (como un interés común) salvo cuando una calamidad grande y común los une, los hace olvidar el respeto y los anima a buscar sus necesidades con fuerza armada; y así algunas veces se enfrentan a los ricos y avanzan sobre todo como una avalancha ${ }^{44}$.

En primer lugar este texto podría sugerir una interpretación restringida del requisito de anexión de la propiedad. Así, los trabajadores al carecer del tipo de propiedad relevante, la tierra, no tendría ninguna clase de derecho político ${ }^{45}$. Sin embargo, como es patente, esto choca con la tesis de que la actividad política específica de los trabajadores es la resistencia ${ }^{46}$, lo cual sería imposible de derecho si estos no formaran parte del cuerpo político. Es posible que en una interpretación débil del requisito de anexión de propiedad este texto cobre más sentido. En efecto, si el trabajador tiene alguna propiedad que anexar al Estado pero, por ejemplo, es incapaz de pagar los suficientes impuestos como para hacerse representar en la cámara de los comunes, todavía es plausible que puedan ejercer el derecho a resistir un gobierno. Esta última interpretación también es consistente con el texto previamente citado On Allegiance and the Revolution, en tanto el consentimiento de una gran masa de la población, que incluso puede tomar las armas, parece necesaria para mantener la estabilidad del país.

\section{Conclusión}

\footnotetext{
${ }^{44}$ J. LOCKE, "Some Considerations of The Consequences of the Lowering of Interest, and Raising the Value of Money", en J. LOCKE, Works of John Locke, Vol, 4., Londres, Rivintgon.

${ }^{45} \mathrm{Si}$ bien el texto parece apuntar, más bien, a que la capacidad racional de los trabajadores se ve impedida por el tipo de tareas que desarrollas, antes que a una cuestión de derecho.

${ }^{46}$ Como también señala J. S. CHUMBITA, «La configuración del pueblo en la resistencia. Un abordaje comteporáneo sobre la obra de John Locke», 196.
} 
A lo largo de este trabajo hemos intentado examinar el concepto lockeano de pueblo a partir de la distinción entre dos tipos de consentimiento. No hemos pretendido saldar la cuestión respecto al auténtico pensamiento de Locke con respecto a la cuestión del pueblo, si es que acaso lo hay. Tal investigación requeriría muchas más precisiones conceptuales y una profundización en el amplio corpus bibliográfico del autor. De manera mucho más modesta, el objetivo de este aporte ha sido señalar algunos matices e indicar caminos de lectura posibles.

Una vez que distinguimos entre los dos tipos de consentimiento y los deberes asociados a cada uno de ellos, es posible sugerir que aquéllos asociados al consentimiento expreso son fecundos para caracterizar a los individuos que pueden formar parte del cuerpo político. En sentido contrario, el deber de obedecer las leyes de un gobierno que es correlativo al consentimiento tácito no aporta tantos matices de interpretación. De ahí que los análisis exclusivamente centrados en hacer consistente la noción de consentimiento que tienen cómo única referencia ese tipo de deberes no contribuyan, tampoco, a la caracterización del cuerpo político.

En particular, los deberes asociados al consentimiento expreso abren un amplio campo de análisis conceptual. Consideramos que, en toda interpretación débil del requisito de anexión de propiedad al Estado, es posible encontrar lugar para realizar un análisis más fecundo de la evidencia textual lockeana. En particular, insistimos en que es posible que Locke haya intentado fundamentar un cuerpo político inclusivo, en tanto toda persona tendría alguna propiedad que anexar al Estado, pero con derechos políticos (en particular, de representación) restringidos a la capacidad impositiva de cada individuo. Esta lectura hace posible sostener que mientras que Locke sostenía una concepción restringida de la participación política parlamentaria y, en consecuencia, el poder político se encontraba limitado, no sucedía lo mismo con el poder constituyente. En efecto, si el cuerpo político posee una base inclusiva, aunque no sea vea representado en su totalidad en el parlamento, sí tiene un rol preponderante en constituir aquélla mayoría que juzga todo intento de resistencia frente al gobierno. Y si bien el derecho resistencia no agota el campo de acción político, no es un tipo de intervención que sea, por eso, despreciable. 